\section{MS39-O2 Hierarchical description of the structural disorder in doped ceria compounds via real and reciprocal space analysis}

Michela Brunelli ${ }^{1}$, Mauro Coduri ${ }^{2}$, Marco Scavini ${ }^{3,4}$, Mattia Allieta $^{3}$, Paolo Masala ${ }^{3}$, Claudio Ferrero ${ }^{5}$

1. Swiss-Norwegian Beamlines, ESRF, BP 220, Grenoble, 38043, France

2. National Research Council, Institute for Energetics and Interphases, Corso Promessi Sposi 29, 23900 Lecco, Italy

3. Università degli Studi di Milano, via Golgi 19, 20133 Milano, Italy

4. CNR-ISTM, via Golgi 19, 20133 Milano, Italy

5. European Synchrotron Radiation Facility, 6 av. J. Horowitz, BP 220, 38043 Grenoble, France

email: brunelli@esrf.fr

In this work, a hierarchical approach for elucidating the structural disorder in doped ceria compounds at different scale lengths is presented.

In doped ceria compounds $\left(\mathrm{Ce}_{1-\mathrm{x}} \mathrm{RE}_{\mathrm{x}} \mathrm{O}_{2-\mathrm{x} / 2}\right.$, with $\mathrm{RE}=\mathrm{La}, \mathrm{Nd}, \mathrm{Sm}, \mathrm{Gd}, \mathrm{Y}, \mathrm{Yb}$ ) ionic conductivity occurs by migration through the oxygen vacancies introduced for charge balance, after doping with lower valent cations. Oxygen diffusion is though impeded above a critical dopant concentration which is likely to originate from defects aggregations.

The aim of this investigation is to describe the structure of such defect aggregations, by following the structural evolution of these materials at different length scales: from the short to the long-range via the analysis of the disorder at the mesocopic scale. This is achieved by combining real space (Pair Distribution Function) and reciprocal space (Rietveld refinement and the line profile analysis) analysis of powder diffraction data.

The relationships between the fluorite structure of ceria $\left(\mathrm{CeO}_{2}\right)$ and the $\mathrm{C}$-type structure of the dopant oxides was exploited: the structure of doped samples is described with the structure of the C-type using one structural disorder parameter (the positional degree of freedom of cations). The reciprocal space analysis showed that doped samples undergo a phase transition from fluorite to C-type at $\sim 0.3$ doping concentration, while at the local scale a continuum of structural evolution is evident: dopant-rich- and Ce-rich droplets, (i.e. small regions, few Ångstroms wide) form and coexist, exhibiting either a distorted fluorite or a C-type structure in the entire compositional range. At larger length scale these droplets average into C-type nanodomains embedded in the fluorite matrix, whose extension increases with doping. The forced connection of these nanodomains led to the formation of antiphase boundaries, observed as peak broadening in the $Q$-space patterns of superstructure peaks only ${ }^{1}$.

Furthermore, we introduce a more general crystallographic rationale to describe the fluorite to C-type phase transformation mechanism, which is based on the percolation of hierarchical defect structures. The approach could be generally applied for the analysis of disorder in other highly doped materials.

\section{Reference:}

${ }^{1}$ M. Coduri, M. Scavini, M. Allieta, M. Brunelli and C. Ferrero, Chem. Mater. 25, 4278-4289 (2013)

Keywords: Doped Ceria, Disorder, Pair Distribution Function; Powder Diffraction, Hierarchy 